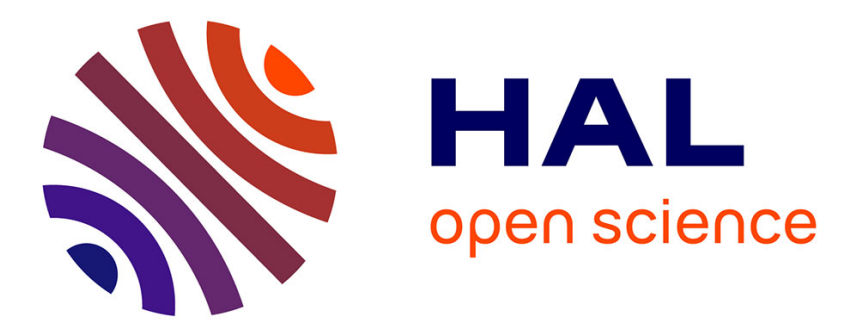

\title{
Nonhydrostatic stabilization of an orthorhombic phase of zirconia
}

\author{
Giuseppe Fadda, Lev Truskinowsky, Giovanni Zanzotto
}

\section{To cite this version:}

Giuseppe Fadda, Lev Truskinowsky, Giovanni Zanzotto. Nonhydrostatic stabilization of an orthorhombic phase of zirconia. Physical Review B: Condensed Matter and Materials Physics (19982015), 2003, 68 (13), 10.1103/PhysRevB.68.134106 . hal-00111394

\section{HAL Id: hal-00111394 \\ https://hal.science/hal-00111394}

Submitted on 29 Jul 2019

HAL is a multi-disciplinary open access archive for the deposit and dissemination of scientific research documents, whether they are published or not. The documents may come from teaching and research institutions in France or abroad, or from public or private research centers.
L'archive ouverte pluridisciplinaire HAL, est destinée au dépôt et à la diffusion de documents scientifiques de niveau recherche, publiés ou non, émanant des établissements d'enseignement et de recherche français ou étrangers, des laboratoires publics ou privés. 


\title{
Nonhydrostatic stabilization of an orthorhombic phase of zirconia
}

\author{
Giuseppe Fadda, ${ }^{1, *}$ Lev Truskinovsky, ${ }^{2,1, \dagger}$ and Giovanni Zanzotto ${ }^{3, \$}$ \\ ${ }^{1}$ Department of Aerospace Engineering and Mechanics, University of Minnesota, 110 Union St. SE, Minneapolis, Minnesota 55455, USA \\ ${ }^{2}$ Laboratoire de Mécanique des Solides, CNRS-UMR 7649, Ecole Polytechnique, 91128 Palaiseau, France \\ ${ }^{3}$ Dipartimento di Metodi e Modelli Matematici per le Scienze Applicate (DMMMSA), Università di Padova, Via Belzoni 7, \\ 35131 Padova, Italy
}

(Received 22 April 2003; published 8 October 2003)

\begin{abstract}
An explicit polynomial strain-energy function for tetragonal-orthorhombic-monoclinic zirconia $\left(\mathrm{ZrO}_{2}\right)$, calibrated from the conventional hydrostatic $p-T$ phase diagram, is used to study the effects of nonhydrostatic loading on the phase equilibria in this material. Several representative sections of the phase diagram of $\mathrm{ZrO}_{2}$ in temperature and stress space, containing both triple and critical points, are computed. A new orthorhombic structure of $\mathrm{ZrO}_{2}$ is predicted to be the most stable phase for a variety of experimentally accessible shear loads, in a wide range of temperatures and pressures.
\end{abstract}

DOI: 10.1103/PhysRevB.68.134106

PACS number(s): $61.50 . \mathrm{Ks}, 62.20 .-\mathrm{x}, 81.30 . \mathrm{Kf}$

\section{INTRODUCTION}

The toughness of brittle ceramics can be increased by an order of magnitude through the introduction of small transforming inclusions that undergo irreversible phase changes under severely nonhydrostatic and mostly tensile stresses, near the tip of a propagating crack. ${ }^{1}$ One of the most important toughening agents in such transformation-toughened ceramics is zirconia $\left(\mathrm{ZrO}_{2}\right),{ }^{2,3}$ and the relevant domain of the $p-T$ phase diagram of this material, which includes the tetragonal, orthorhombic (ortho I), and monoclinic phases, has been investigated experimentally in Refs. 4-8. A comprehensive Landau strain-energy function providing a unified thermodynamical description of these $\mathrm{ZrO}_{2}$ phases under hydrostatic conditions has been constructed in Refs. 9 and 10. The hydrostatic phase diagram, however, is not sufficient for the analysis of the behavior of the zirconia inclusions near the tip of a crack, where the stresses exhibit strong shear components. The transformation conditions allowing one to compute the crack-shielding and toughness-increase effects must instead be formulated as a "transformation criterion" in stress space. ${ }^{11,12}$ The problem is then to determine the phase equilibria of zirconia under nonhydrostatic loads. The overall effect of the latter on the temperatures of the relevant phase transitions is expected to be much stronger than the influence of hydrostatic pressure since, for instance, the shear effect of the well-known tetragonal-to-monoclinic phase transformation in $\mathrm{ZrO}_{2}$ is about four times larger than the corresponding volumetric effect. Experimental measurements in uniaxial and bending tests for this system are quite limited and are available only for stabilized configurations of tetragonal $\mathrm{ZrO}_{2} \cdot{ }^{13-15}$ It has been observed, however, that the relatively high plasticity limit in this material permits a purely elastic analysis of its nonhydrostatic phase equilibria. ${ }^{16}$

To organize the existing data and focus future experimental research on nonhydrostatic equilibria, in this paper we employ the phenomenological model of Ref. 10 to study the effects of various shear loads on $\mathrm{ZrO}_{2}$ crystals. We compute a number of experimentally relevant sections of the full stress-temperature phase diagram and make predictions concerning the stability domains of the zirconia phases for a variety of nonhydrostatic conditions, demonstrating the strong effect of the shear stresses on the phase equilibria observed under hydrostatic conditions. We also show the presence of new triple points in the temperature-stress phase diagrams and of critical points where severely sheared phases, which possess different symmetries under hydrostatic loads, become indistinguishable. ${ }^{17}$ The most interesting prediction is that a new orthorhombic zirconia structure, already shown in Ref. 10 to have a small metastability range at high temperatures and low pressures, is the most stable one for a variety of shear loads, also at room conditions; this new phase may potentially become dominant in the vicinity of a crack tip. The new structure can only be partially characterized by our model, and requires the use of suitable crystallographic (see Appendix B) and ab initio methods for a complete study. In addition to $\mathrm{ZrO}_{2}$, our approach and results may prove useful also for a better understanding of the behavior of other oxides, such as hafnia $\mathrm{HfO}_{2}$.

This paper is organized as follows. After giving brief crystallographic preliminaries and an introduction to our strain energy function in Sec. II, we study in Sec. III the behavior of zirconia crystals under hydrostatic or uniaxial, compressive or tensile loads. The calculated phase diagrams confirm that the nonisotropic loads have a marked effect on the known phase equilibria of $\mathrm{ZrO}_{2}$, including the tetragonal-to-monoclinic transformation. We also find that the new orthorhombic phase mentioned above gains stablility in hydrostatic tension, or uniaxial compression, over a wide range of temperatures. In Sec. IV we study the behavior of zirconia crystals under shear loads that may distort all the $\mathrm{ZrO}_{2}$ structures considered here, converting the symmetryrelated variants of the low-symmetry configurations into independent phases. In this case we find that the monoclinic phase is stabilized (versus the tetragonal one) at high temperatures for low shears, while for higher shears and temperatures the new orthorhombic structure again becomes the most stable one. We also calculate a section of the sheartemperature diagram at higher pressure, which exhibits, at relatively small shears, symmetric critical points for the tetragonal and ortho I phases. In the Appendixes we provide some additional details regarding our ${ }^{10}$ Landau energy for 


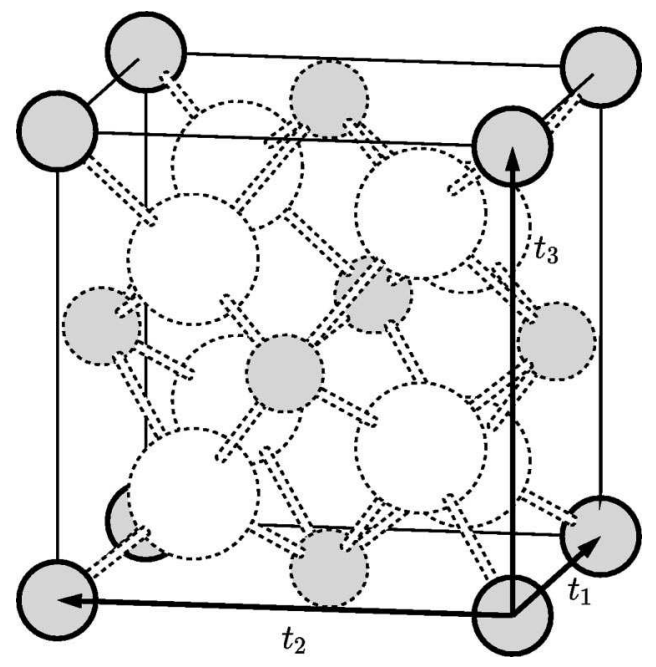

FIG. 1. The primitive-tetragonal reference cell with $Z=4$ ( $Z$ being the number of chemical units), used to describe the deformations of the $\mathrm{ZrO}_{2}$ structures; $\mathrm{Zr}$ atoms are in gray, $\mathrm{O}$ atoms in white. This cell is spanned by the basis vectors $t_{a}, a=1,2,3$, with the fourfold axis along $t_{3}$; the dashed atoms of the zirconia crystal are disregarded in this model. The order parameters $y_{3}$ and $y_{6}$ break the equality of the lengths of the vectors $t_{1}$ and $t_{2}$ and their orthogonality condition, respectively (see Appendix A).

$\mathrm{ZrO}_{2}$, and briefly discuss some possibilities for the crystallographic structure of the new orthorhombic phase that is stable under nonhydrostatic loads.

\section{TRANSFORMATION MECHANISM, ORDER PARAMETERS, AND ENERGY FUNCTION}

In the area of the $p$ - $T$ phase diagram not too far from room conditions, zirconia exhibits the following three phases: tetragonal $(t)$, orthorhombic "ortho I" (o1), and monoclinic $(\mathrm{m})$, with a triple point located at about $840 \mathrm{~K}$ and $1.8 \mathrm{GPa}$. References $4-8$ and $18-21$ give the space groups and detailed crystallographic descriptions of these and other zirconia polymorphs, and present the experimental phase diagrams (see also Ref. 10 for some details). If certain atoms are disregarded (see Fig. 1), the $t-o 1-m$ structures of $\mathrm{ZrO}_{2}$ can all be described as originating from the distortion of a primitive tetragonal "skeletal" Bravais lattice, spanned by three mutually orthogonal basis vectors $t_{1}, t_{2}$, and $t_{3}$.

In our simplified setting which only considers the skeletal atoms, the symmetry of a phase is dictated by the point group of the corresponding structure. The point group $\mathcal{T}_{3}$ of the $t$ phase is

$$
\mathcal{T}_{3}=\left\{1, R_{t_{1}}^{\pi}, R_{t_{2}}^{\pi}, R_{t_{3}}^{\pi}, R_{t_{1}+t_{2}}^{\pi}, R_{t_{1}-t_{2}}^{\pi}, R_{t_{3}}^{\pi / 2}, R_{t_{3}}^{3 \pi / 2}\right\}
$$

where $R_{k}^{\psi}$ denotes a rotation of angle $\psi$ about the axis $k$, and only the elements with positive determinant are listed. From an analysis of the literature,,$^{2,6,18-25}$ we conclude ${ }^{9,10}$ that the $o 1$ phase has point group

$$
\mathcal{O}_{123}=\left\{1, R_{t_{1}}^{\pi}, R_{t_{2}}^{\pi}, R_{t_{3}}^{\pi}\right\}
$$

while (since the fourfold axis of the $t$ phase is parallel to the twofold axis of the $m$ phase) the $m$ phase has point group

$$
\mathcal{M}_{3}=\left\{1, R_{t_{3}}^{\pi}\right\}
$$

The orientation relationships given by Eqs. (1)-(3) are also confirmed by Ref. 3. The (sub)groups in Eqs. (2) and (3) determine the form of the strains that produce, from the tetragonal skeletal lattice in Fig. 1, the $o 1$ and $m$ lattices with point groups as above; see Refs. 9, 10, and 26 for details.

For each temperature $T$, we consider as the reference state the tetragonal equilibrium configuration at that temperature (thermal-expansion data for the $t$ phase can be found in Refs. 27 and 28). Correspondingly, we denote by $e_{I}, I$ $=1, \ldots, 6$, the components of the finite strain tensor, indexed as usual according to the Voigt convention (see Refs. 26,29 , and 30). Due to the tetragonal symmetry of the parent lattice, another set of strain coordinates $y_{I}, I=1, \ldots, 6$, proves more convenient in the ensuing analysis: $:^{9,10}$

$$
\begin{gathered}
y_{1}=e_{1}+e_{2}+e_{3}, \quad 6 y_{2}=e_{1}+e_{2}-2 e_{3}, \\
\sqrt{2} y_{3}=e_{1}-e_{2}, \quad y_{4}=e_{4}, \quad y_{5}=e_{5}, \quad y_{6}=e_{6} .
\end{gathered}
$$

The strain components $y_{4}$ and $y_{5}$ are not involved in the transformation mechanism of the zirconia polymorphs discussed here, and will be considered as completely decoupled. The components $y_{1}$ and $y_{2}$ describe the symmetry-preserving stress-free thermal expansion of the tetragonal lattice in Fig. 1 and do not affect the symmetries of the phases under consideration. The remaining strains $y_{3}$ and $y_{6}$ are the order parameters in our model (see Fig. 1): conditions $y_{3}=y_{6}=0$ select the $t$ phase (with point group $\mathcal{T}_{3}$ ); conditions $y_{3} \neq 0$ and $y_{6}=0$ give the $o 1$ phase (with point group $\mathcal{O}_{123}$ ); finally, for $y_{3}$ and $y_{6}$ both nonzero we obtain the $m$ phase (with point group $\mathcal{M}_{3}$ ). The configurations with $y_{6} \neq 0$ and $y_{3}=0$ identify a second orthorhombic phase (o2) with point group

$$
\mathcal{O}_{1 \pm 2,3}=\left\{1, R_{t_{3}}^{\pi}, R_{t_{1}+t_{2}}^{\pi}, R_{t_{1}-t_{2}}^{\pi}\right\}
$$

The theory of Ref. 10 indicates that such an $o 2$ phase, although not reported in the experimental literature, has a small metastability range for high temperatures and low pressures. As we show below, under suitable nonhydrostatic loadings this range is extended, making $o 2$ the most stable structure for $\mathrm{ZrO}_{2}$ in a wide domain of pressures and temperatures. $^{31}$

We attribute to any homogeneous deformation of the reference configuration in Fig. 1 a tetragonally invariant free energy density $\phi$ per unit reference volume, which depends on $y_{1}, \ldots, y_{6}$ and $T$. A suitable polynomial expansion for $\phi$ has been derived in Ref. 10 and is recalled in Appendix A. To obtain the Gibbs free energy density $\phi_{G}$ we add to $\phi$ the potential of a general nonhydrostatic load:

$$
\phi_{G}\left(y_{I}, \bar{\sigma}_{I}, T\right)=\phi\left(y_{I}, T\right)-\sum_{J=1}^{6} \bar{\sigma}_{J} y_{J}
$$


$(I=1, \ldots, 6)$, where the stress components $\bar{\sigma}_{I}$, referred to the same basis as the strains $y_{I}$, are related as follows to the usual stress coordinates $\sigma_{I}$ conjugate to the strains $e_{I}$ :

$$
\begin{gathered}
\bar{\sigma}_{1}=-p=-\left(\sigma_{1}+\sigma_{2}+\sigma_{3}\right) / 3 \\
\bar{\sigma}_{2}=\sigma_{1}+\sigma_{2}-2 \sigma_{3}, \quad \bar{\sigma}_{3}=\left(\sigma_{1}-\sigma_{2}\right) / \sqrt{2} \\
\bar{\sigma}_{A}=\sigma_{A} \text { for } A=4,5,6 .
\end{gathered}
$$

The Landau potential $\phi_{L}$ is then derived from Eq. (7) through the adiabatic elimination of all deformational variables other than the order parameters $y_{3}$ and $y_{6}$, and since the dependence of $\phi$ on $y_{1}, y_{2}, y_{4}$, and $y_{5}$ in Eq. (6) is assumed to be convex (quadratic), there is a unique solution of the equilibrium equations $\partial \phi_{G} / \partial y_{R}=0$ for $R=1,2,4,5$.

In this paper we limit our attention to loads with $\bar{\sigma}_{4}=0$, $\bar{\sigma}_{5}=0 .{ }^{32}$ Under these conditions, we obtain the function

$$
\begin{aligned}
& \phi_{L}\left(y_{3}, y_{6}, \bar{\sigma}_{1}, \bar{\sigma}_{2}, \bar{\sigma}_{3}, \bar{\sigma}_{6}, T\right) \\
& =\psi\left(y_{3}, y_{6}, \bar{\sigma}_{1}, \bar{\sigma}_{2}, T\right)-\bar{\sigma}_{3} y_{3}-\bar{\sigma}_{6} y_{6},
\end{aligned}
$$

whose explicit form can be found in Eq. (A6) of Appendix A. We notice that, as $\psi$ is an even function of $y_{3}$ and $y_{6}$, the energy $\phi_{L}$ is invariant under the simultaneous reflection of $y_{3}$ and $\bar{\sigma}_{3}$, or of $y_{6}$ and $\bar{\sigma}_{6} \cdot{ }^{33}$ The critical points of $\phi_{L}$ are obtained by solving the equilibrium equations $\partial \phi_{L} / \partial y_{R}=0$ for $R=3,6$. The global phase diagram in the fivedimensional space $\left(\bar{\sigma}_{1}, \bar{\sigma}_{2}, \bar{\sigma}_{3}, \bar{\sigma}_{6}, T\right)$ will then distinguish the domains with different structures of the global minima of $\phi_{L}$, as well as the metastability domains for each minimizer. Since $p=-\bar{\sigma}_{1}$, one obtains the standard Gibbs free energy density $g(p, T)$ for a given phase by setting $\bar{\sigma}_{2}=\bar{\sigma}_{3}=\bar{\sigma}_{6}$ $=0$ in Eq. (8), and minimizing the resulting function with respect to $y_{3}$ and $y_{6}$. The sets of minimizers $\left(y_{3}(p, T), y_{6}(p, T)\right)$ obtained in this way represent the different stable phases under consideration, and give distinct energy functions $g(p, T)$ for the different phases. In the conventional approach such functions are obtained separately through direct modeling, rather than through the elimination of internal parameters; this obscures the fact that each phase is represented by a set of symmetry-related variants. Consideration of such individual "twins," however, is important when the original symmetry relations among the variants are destroyed by shear stresses, and the twins become independent phases. The present approach, which unfolds the complete energy landscape in the original strain variables, allows one to take these effects into consideration and trace the connections among all the energy wells.

\section{LOADS CONJUGATE TO NON-ORDER-PARAMETERS}

In this section we study how phase equilibria in zirconia are affected by the loads $p=-\bar{\sigma}_{1}$, representing hydrostatic pressure or tension, and $\sigma_{3}$, which is responsible for uniaxial tension or compression along the highest-symmetry axis $t_{3}$ in Fig. 1. A more natural variable for this non-hydrostatic load is

$$
\tau=-\left(2 \bar{\sigma}_{1}+\bar{\sigma}_{2} / 3\right)=\sigma_{3}+p
$$

which is negative for uniaxial compression and positive for uniaxial tension (in excess of the isotropic load $p$ ). As is the case for $p$, the application of $\tau$ may affect the $c / a$ ratio of the $t$ phase and eventually activate the order parameters $y_{3}$ and $y_{6}$, which produce the symmetry-breaking transformation. However, as the strain variables conjugate to $p$ or $\tau$ are orthogonal to $y_{3}$ and $y_{6}$ [see Eqs. (4), (7), and (9)], $p$ and $\tau$ may activate the order parameters only through the higherorder coupling terms involving $y_{3}$ and $y_{6}$ in Eq. (6); therefore these two types of loading do not change the symmetry groups considered in Sec. II, as the equilibrium equations and the corresponding solutions maintain their original invariance for all $p$ and $\tau$ (also the variant structures for the low-symmetry configurations are preserved). We notice that for this reason the corresponding phase diagrams are not symmetric in tension or compression, i.e., under a change of sign for $p$ or $\tau$. In each diagram we use the strain energy (8) to obtain quantitative information on the corresponding phase relations (see Appendix A for the explicit energy function). In addition to the standard equilibrium phase boundaries ("Maxwell lines," indicated by thick lines in the figures), in the computed phase diagrams hereafter we present also the boundaries of the stability domains of the different phases, which indicate the combinations of parameters where the corresponding matrix of elastic moduli softens (dashed lines).

The role of compressive hydrostatic pressure $p>0$ is described by the conventional thermodynamic $p-T$ diagram, which in Ref. 10 was calibrated from experimental data. Here we extend our previous analysis by covering also the domain of hydrostatic tension $p<0$ (see Fig. 2), which is relevant for the description of phase transformations near the tip of a crack. The most interesting feature of this extended diagram is the appearance, at high temperatures and high tensile loads (important for transformation toughening), of the new orthorhombic phase $o 2$ with symmetry of $\mathcal{O}_{1 \pm 2,3} \cdot{ }^{34}$ For $p=-0.16 \mathrm{GPa}, T=1560 \mathrm{~K}$, we obtain a new $t-o 2-m$ triple point, where the three Maxwell lines, numbered 1 $(t-m), 3(t-o 2)$, and $4(m-o 2)$ meet. This triple point must be distinguished from the well-known triple point with coexisting $t, o 1$, and $m$ phases, studied in Ref. $10 .{ }^{35}$ We observe that around each triple point there is an extended domain where three metastable phases coexist. Since such domains do not overlap, the possibility of four coexisting metastable phases, although theoretically allowed for a one-component system, is ruled out by the numerical values of the Landau coefficients suitable for $\mathrm{ZrO}_{2}$ (see Table I).

Notice that the equilibrium phase boundaries in Fig. 2 separating the domain of absolute stability of the phase $o 2$ have negative slopes. This is a result of the negative volumetric effect of the transitions $m-o 2(\Delta V / V=-0.19 \%$ from $m$ to $o 2)$ and $o 2-t(\Delta V / V=-4.67 \%$ from $o 2$ to $t)$. We remark that the conventional orthorhombic phase $o 1$ has almost the same density as the $t$ phase, with $\Delta V / V=-1.5 \% .^{2}$ 


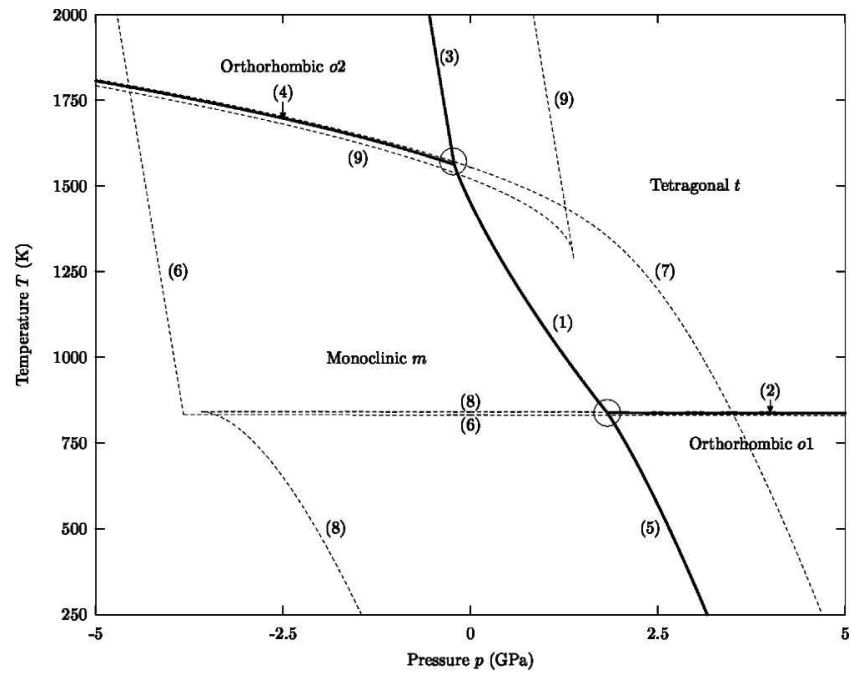

FIG. 2. Calculated phase diagram of zirconia in the $(p, T)$ plane. The $t-m, t-o 1, t-o 2, m-o 2$, and $m-o 1$ Maxwell lines (boundaries of absolute phase stability) are the thick solid lines numbered 1 to 5 , respectively. The limits of stability for the $t, m, o 1$, and $o 2$ phases, marked by dashed lines, are, respectively, within lines 6 , below line 7 , within lines 8 , and within lines 9. The $t-o 1-m$ and $t-o 2-m$ triple points are marked by circles (only the former lies in the half-plane $p>0$ that is usually considered).

Such a low volumetric effect, which makes the corresponding phase boundary almost parallel to the pressure axis, probably contributed to the early confusion, in the experimental literature, between the $t$ and $o 1$ zirconia phases.

In Fig. 3 we show the $\tau-T$ section for $p=0$ of the global three-dimensional $\tau-p-T$ phase diagram. Here the $t, o 2$, and $m$ phases are the absolute minimizers in their respective domains, with the standard $o 1$ configuration present only as a metastable phase (relative minimizer), below line 8 . The three equilibrium Maxwell lines, numbered $1(t-m), 3$ $(t-o 2)$, and $4(m-o 2)$, meet at a $t-o 2-m$ triple point, located at about $1400 \mathrm{~K}$ and $-85 \mathrm{MPa}$. The fact that the nonisotropic load $\tau$ preserves the structure of the symmetry-related phase variants, which all maintain equal energy, is illustrated by the energy profile in the lower part of Fig. 3. We observe that the effect of uniaxial tension $(\tau>0)$ is to raise the $t-m$ transformation temperature, enlarging the stability domain of the $m$ phase; we obtain for the derivative $d T / d \tau$ along the $t-m$ phase boundary an average value of $1010 \mathrm{~K} \mathrm{GPa}^{-1}$.
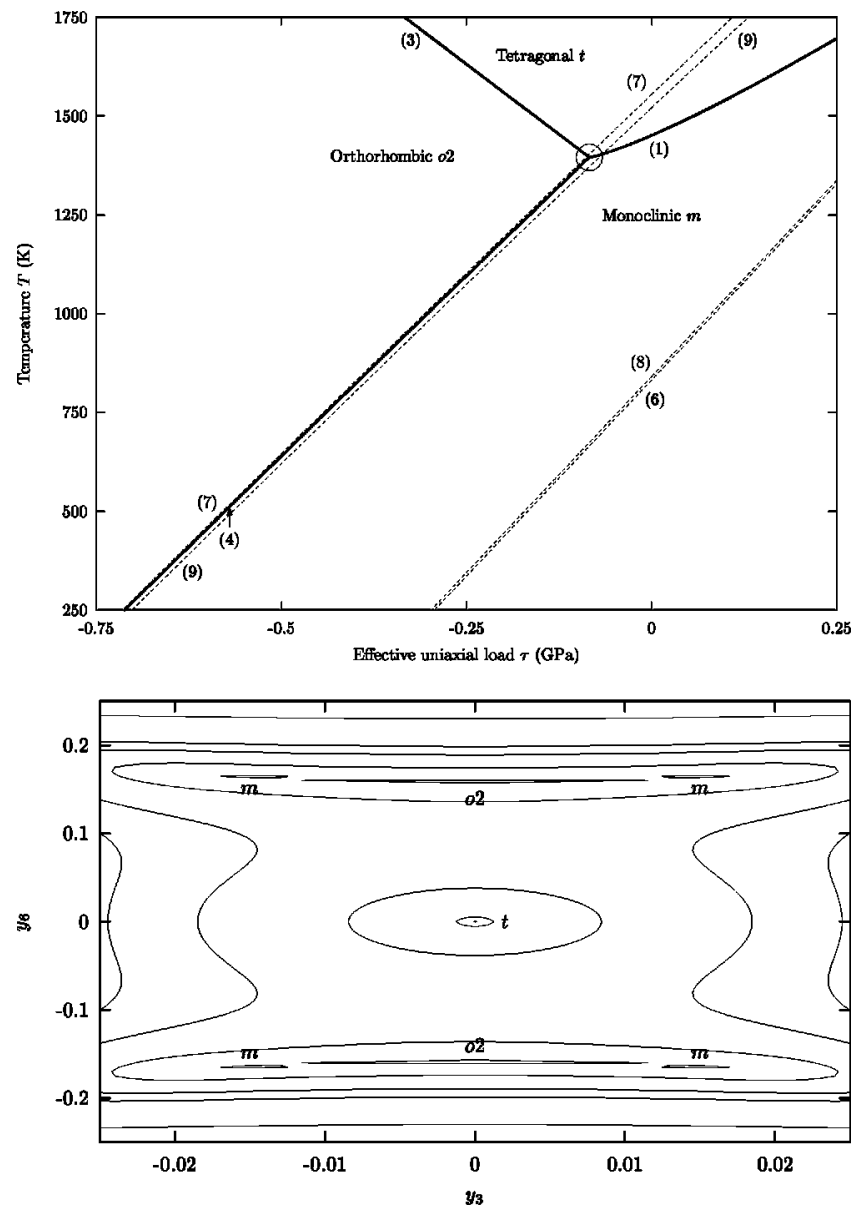

FIG. 3. Calculated phase diagram of zirconia at room pressure in the $(\tau, T)$ plane with $\tau=\sigma_{3}+p$. The Maxwell lines (thick solid lines) numbered 1,3 , and 4 meet at a $t-o 2-m$ triple point, marked by a circle. Indicated are also the partially overlapping stability domains for each of the $t, m, o 1$, and $o 2$ phases (marked by dashed lines), which are above line 6 , below line 7 , below line 8 , and above line 9, respectively. The level sets and minimizers of the Landau energy surface at conditions near the triple point are also shown.

For uniaxial compression $\tau<0$, on the other hand, the stability domain of the $t$ phase is at first enlarged, but at higher compressions the new $o 2$ structure becomes the most stable one. This gives in principle a way to detect experimentally such an $o 2$ phase, as uniaxial compression at room

TABLE I. Numerical values of the coefficients in Eqs. (A1)-(A6), calibrated from experimental data in Ref. 10. All quantities are in GPa, except for $A_{3}$ and $A_{6}$ (in $\mathrm{GPa} \mathrm{K}^{-1}$ ), and $T_{0}$ (in $\mathrm{K}$ ).

\begin{tabular}{lllcll}
\hline \hline$A_{3}$ & $3.88 \times 10^{-1}$ & $A_{6}$ & $2.82 \times 10^{-3}$ & $B_{6}$ & $1.18 \times 10^{1}$ \\
$T_{0}$ & $8.32 \times 10^{2}$ & $H_{3}$ & $-5.9 \times 10^{3}$ & $H_{6}$ & $-1.94 \times 10^{5}$ \\
$\bar{C}_{133}$ & $-3 \times 10^{3}$ & $\bar{C}_{233}$ & $8.17 \times 10^{3}$ & $\bar{C}_{166}$ & $-6.13 \times 10^{2}$ \\
$\bar{C}_{266}$ & 5.91 & $\bar{C}_{344}$ & $5.61 \times 10^{1}$ & $\bar{C}_{456}$ & $8.42 \times 10^{1}$ \\
$\widetilde{D}_{3}$ & $-9.66 \times 10^{4}$ & $\widetilde{L}$ & $-1.08 \times 10^{4}$ & $\widetilde{D}_{6}$ & $-2.45 \times 10^{3}$ \\
$K_{3}$ & $6.66 \times 10^{8}$ & $K_{6}$ & $7.29 \times 10^{4}$ & & \\
$C_{11}$ & 307 & $C_{33}$ & 320 & $C_{44}$ & 100 \\
$C_{66}$ & 16 & $C_{12}$ & 48 & $C_{13}$ & 209 \\
\hline \hline
\end{tabular}




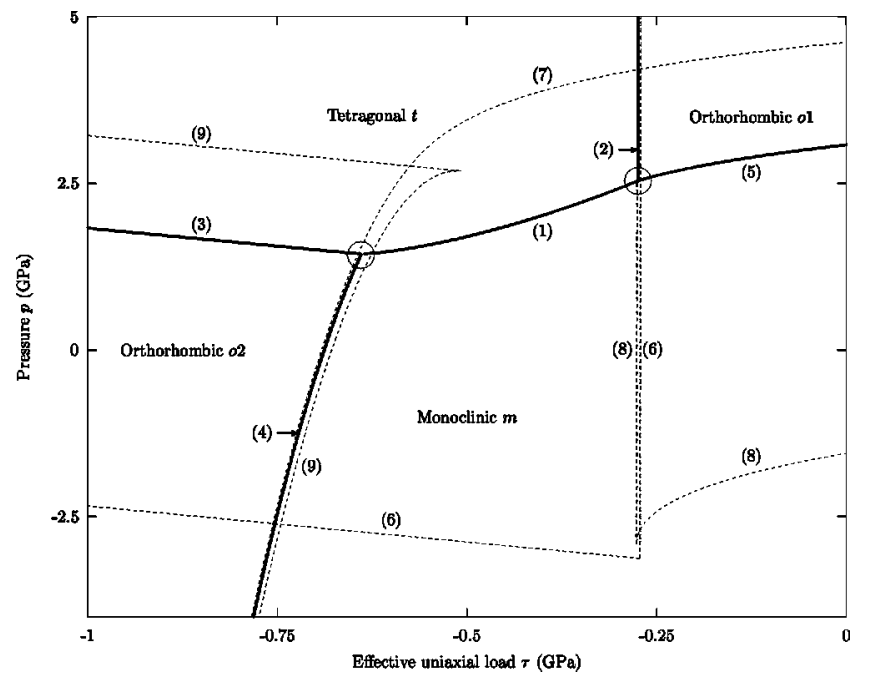

FIG. 4. Calculated phase diagram of zirconia in the $(\tau, p)$ plane at room temperature. Same legend as in Fig. 2.

temperature and pressure should produce a $m-o 2$ transformation in $\mathrm{ZrO}_{2}$ at about $\tau=-0.7 \mathrm{GPa}$.

In Fig. 4 we present another important section of the $\tau-p-T$ phase diagram, representing the $\tau-p$ plane at room temperature $T=293 \mathrm{~K}$. This is a natural choice from the experimental point of view, and some of the $\tau$ - $p$ phase relations for zirconia have indeed been reported in Refs. 13 and 14 for $\mathrm{CeO}_{2}$-stabilized sintered powder samples. Again, also on this plane, we find that all the four structures $t, o 1, o 2$, and $m$ can be absolute minimizers, with both the $t-o 1-m$ and the $t-o 2-m$ triple points. Most of the slopes of the equilibrium phase boundaries here are positive, from which we deduce, for instance, that the transition from $m$ to $t$ leads not only to the reduction of volume but also to the contraction along the tetragonal axis. The corresponding experimental observation on stabilized powders ${ }^{14}$ shows that the $t-m$ transformation pressure indeed increases with uniaxial tension; the slope of the equilibrium phase boundary was experimentally found to be (in our variables) $d \tau / d p=1.31$. Our computations give an average slope of 0.43 , which, while correct in order of magnitude, is in disagreement with the experimental value possibly due to the random orientations of the grains in the experiments on powders, which make the applied uniaxial load not aligned with any particular axis in the crystal.

We remark that a common approach of phenomenological continuum mechanics, which often treats phase transitions as a form of "plastic" deformation, ${ }^{13,14}$ would be to use the result of a uniaxial test to fit parameters within a standard hypothesis for the transformation "yield surface," satisfying general conditions of material symmetry. For instance, the "flow" surface of the Mohr-Coulomb type was constructed in Ref. 13 based on a tensile test, and applied subsequently for the interpretation of a bending test. In contrast, in our approach we are not constrained by any a priori structure of the transformation surface or "flow rule"; moreover, we can distinguish between the equilibrium (Maxwell) phase boundaries and the stability boundaries, indicating the limits of ultimate strength of the corresponding phases. Despite this flexibility, the issue of finding a unifying ansatz for the Maxwell surfaces of $\mathrm{ZrO}_{2}$, representing them in an approximate but simple way in global stress space, remains an interesting challenge.

\section{LOADS CONJUGATE TO ORDER PARAMETERS}

In this section we consider nonhydrostatic loads conjugate to the order parameters $y_{3}$ and $y_{6}$. These shear loads, with components $\bar{\sigma}_{3}$ and $\bar{\sigma}_{6}=\sigma_{6}$, disrupt the structure of the symmetry-related variants because their potential produces a "tilt" of the original energy surface and the equilibrium equations no longer have parity in $y_{3}$ or $y_{6}$. This turns the variants of the orthorhombic and monoclinic structures into independent phases, which are no longer grouped into orbits of equienergetic wells.

Consider first the $\sigma_{6}$ loading. In this case parity in $y_{6}$ is lost, so that the four $m$ solutions with monoclinic point group $\mathcal{M}_{3}$ (which for $\sigma_{6}=0$ only differ by the four possible permutations of signs of $y_{3}$ and $y_{6}$, but are otherwise equivalent) are now split into two independent pairs of variants: $\mathrm{m}^{+}$ for $y_{6}>0$, and $m^{-}$for $y_{6}<0$ (their point group is always $\mathcal{M}_{3}$ ), and analogously for the two $o 2^{+}$and $o 2^{-}$orthorhombic wells (which both have point group $\mathcal{O}_{1 \pm 2,3}$ ). We remark that while the $o 2$ and $m$ energy minimizers preserve their symmetry under the load $\sigma_{6}$, the $t$ minimizer does not maintain its $\mathcal{T}_{3}$ point group, and immediately becomes a "distorted tetragonal" phase (still called $t$ ) with point group $\mathcal{O}_{1 \pm 2,3}$.

The computed $\sigma_{6}-T$ phase diagram at room pressure, which is necessarily symmetric with respect to the reflection about the $T$ axis, is shown in Fig. 5. The two new monoclinic "phases," represented by the two pairs of wells $m^{ \pm}$, maintain the same energy along line 10 in the diagram (i.e., for $\sigma_{6}=0$ ), which may therefore be viewed as an equilibrium (Maxwell) boundary; the same is true for the other two new $o 2^{ \pm}$phases (see the energy plot in the lower part of Fig. 5).

We observe that the overall effect of the $\sigma_{6}$ shear loading on the $t-m$ phase change at room pressure is to raise fairly steeply the transformation temperature for small loads, with a stabilization of the low-symmetry $m$ phase (variants $m^{+}$) to above $1550 \mathrm{~K}$. It is instructive to compare this effect with the corresponding effect of hydrostatic pressure, studied in the preceding section. Our computation here gives $d T / d \sigma_{6}$ $=1660$, which should be compared to $d T / d p=-370$ (all figures in $\mathrm{K} \mathrm{GPa}^{-1}$ ); the large numerical value of the slope of the $t-m$ phase boundary in the $\sigma_{6}-T$ plane and the associated drastic promotion of the $m$ phase achieved through the application of the $\sigma_{6}$ shear load can be attributed to the large transformation shear strain (17\%), compared to the volumetric effect $(5 \%)$, in this phase change. Notice also that, contrary to the case of classical plasticity, the "yield stress" for transformation plasticity in tetragonal zirconia increases with temperature, rather than decreases. In Fig. 5 we further notice that at higher shear stresses and temperatures, the $m$ phase, initially promoted by the $\sigma_{6}$ load, again gives way to the new orthorhombic phase $o 2$ (variant $o 2^{+}$). The phase diagram in Fig. 5 thus exhibits three triple points: 

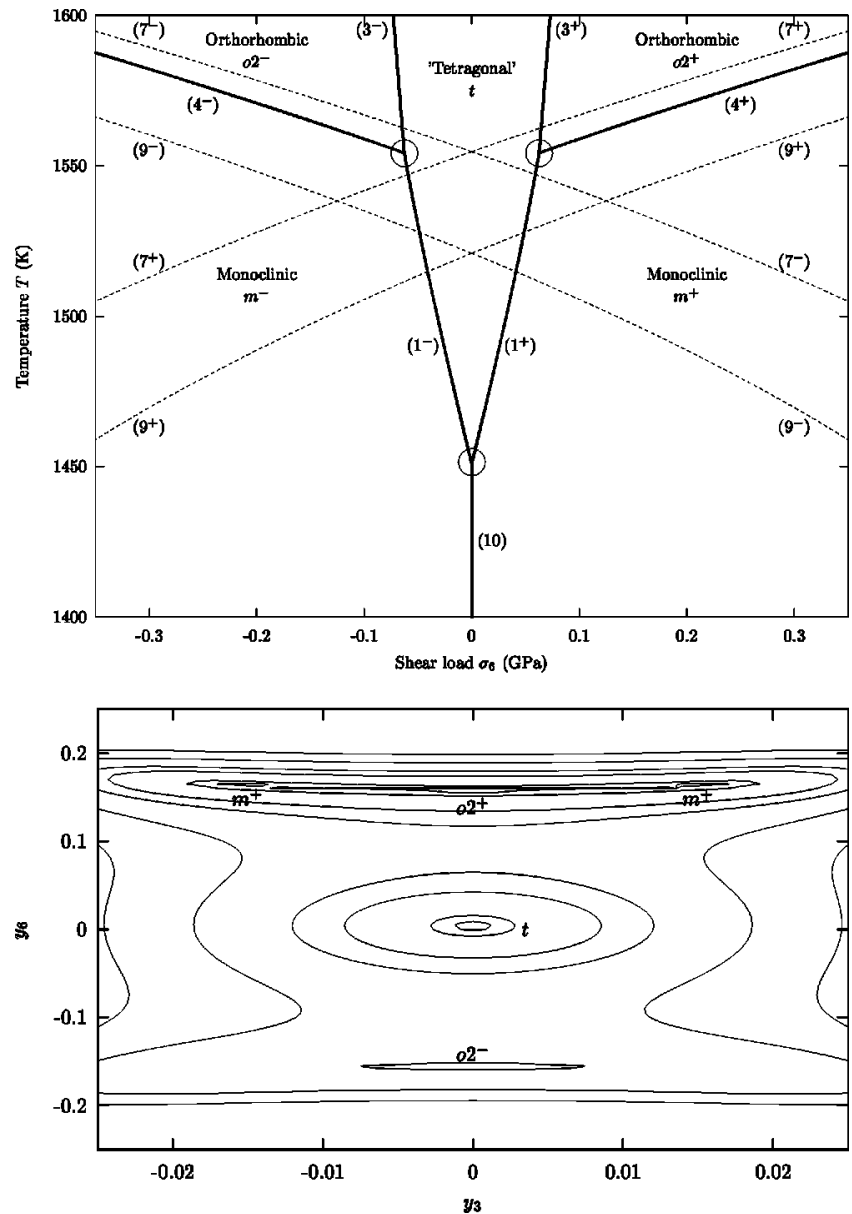

FIG. 5. Calculated phase diagram of zirconia in the $\left(\sigma_{6}, T\right)$ plane at room pressure. For clarity only the portion for $T$ $>1400 \mathrm{~K}$ is shown. The Maxwell lines are the thick solid lines numbered $1^{ \pm}\left(t-m^{ \pm}\right), 3^{ \pm}\left(t-o 2^{ \pm}\right), 4^{ \pm}\left(o 2^{ \pm}-m^{ \pm}\right)$, and 10 $\left(m^{+}-m^{-}\right)$; the latter is the equilibrium line between the two pairs of monoclinic variants $m^{ \pm}$. The partially overlapping stability domains for $m^{ \pm}$are, respectively, below dashed lines $7^{ \pm}$, and, for $o 2^{ \pm}$, they are above dashed lines $9^{ \pm}$. The triple points are marked by circles; the two upper triple points refer to the equal value of the Landau potential of the distorted-tetragonal $t$, the $o 2^{ \pm}$, and the two $m^{ \pm}$energy wells (all taken with the same + or - sign). The lower triple point refers to the equal depth of the $t, \mathrm{~m}^{+}$, and $\mathrm{m}^{-}$wells for $\sigma_{6}=0$. The level sets and minimizers of the "tilted" Landau potential for conditions near the $t-o 2^{+}-m^{+}$triple point are shown below the phase diagram; notice the absence of the variants $\mathrm{m}^{-}$, which at these conditions (above line $7^{-}$) are unstable.

$t-o 1^{+}-m^{+}, t-o 1^{-}-m^{-}$, and $t-m^{+}-m^{-}$. Two of these triple points correspond to conventional $t-o 2-m$ three-phase equilibria, while the third one corresponds to the coexistence of the $t$ and $m$ structures, the $m$ phase being represented by two sets of $m^{-}$and $m^{+}$twins. The latter are crystallographically and energetically equivalent at $\sigma_{6}=0$, but become distinct as the energy landscape gets tilted for any nonzero value of $\sigma_{6}$.

One can speculate that for higher $T$ and higher (positive or negative) $\sigma_{6}$ the $t-o 1^{+}$and $t-o 1^{-}$phase boundaries may terminate at critical points where the difference between the corresponding phases disappears. We did not extend to such conditions the computations reported in Fig. 5, because the

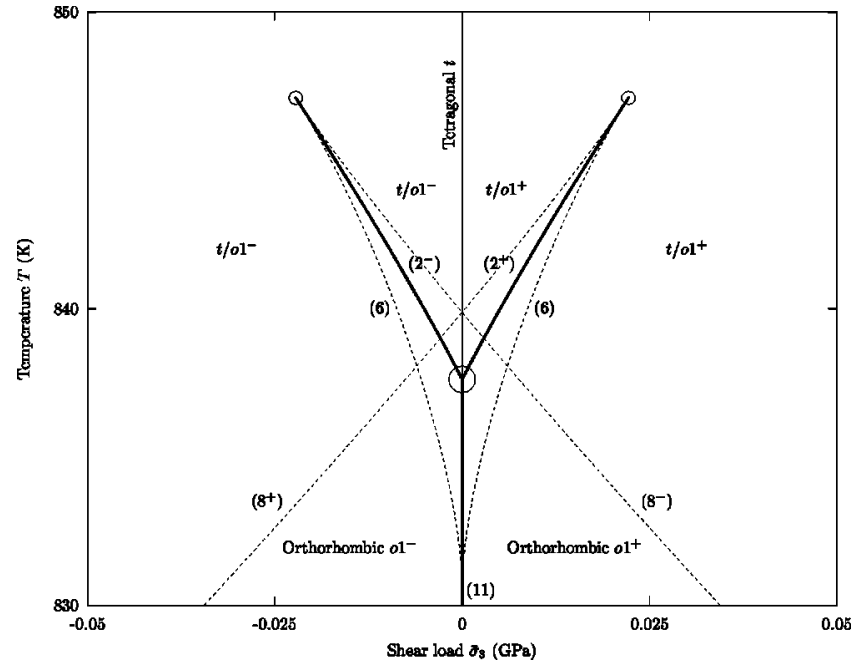

FIG. 6. Calculated phase diagram of zirconia in the $\left(\bar{\sigma}_{3}, T\right)$ space, for $p=4 \mathrm{GPa}$. The Maxwell lines are the thick solid lines numbered $2^{ \pm}\left(t-o 1^{ \pm}\right)$, and $11\left(o 1^{+}-o 1^{-}\right)$, where the two independent variants of the $o 1$ phase (with orthorhombic $\mathcal{O}_{123}$ symmetry) are denoted by $o 1^{+}$and $o 1^{-}$according to the sign of $y_{3}$ (the undistorted $t$ phase with symmetry $\mathcal{T}_{3}$ only exists for $\bar{\sigma}_{3}=0$, while for $\bar{\sigma}_{3} \neq 0$ it has $\mathcal{O}_{123}$ symmetry). The stability domain is above the dashed lines number 6 for the (distorted) $t$ phase, and below the dashed lines $8^{ \pm}$for $o 1^{ \pm}$phases. The triple point marked by the larger circle refers to the equal depth of the $t-o 1^{+}-o 1^{-}$energy wells. The critical points are marked by smaller circles. The symbols $t / o 1^{ \pm}$indicate that only one distorted structure is stable in the domain outside the "spikes" ending at the two critical points.

presence of the cubic energy well of $\mathrm{ZrO}_{2}$, not taken into account in our model, would considerably affect the hightemperature part of the $\sigma_{6}-T$ diagram.

To illustrate nonetheless the notion of such shear-induced critical points (see also Refs. 11 and 36) we consider the load $\bar{\sigma}_{3}$, which is conjugate to the strain $y_{3}$, related to the $t-o 1$ phase transition. Figure 6 presents a portion of the computed $\bar{\sigma}_{3}-T$ phase diagram for $p=4 \mathrm{GPa}$. Here the only stable phases are $t$ and $o 1$, whose two variants, according to the sign of $y_{3}$, are denoted $o 1^{+}$and $o 1^{-}$; the latter are equivalent symmetry-related twins for $\bar{\sigma}_{3}=0$, and become independent phases under nonzero $\bar{\sigma}_{3}$ loads. The triple point in Fig. 6 refers to the conditions producing the equal depth of such $t, o 1^{+}$, and $o 1^{-}$energy wells. The application of the shear load again significantly extends the stability domain of the low-symmetry phase $o 1$, as it strongly affects the temperature of the $t-o 1$ phase change: the slope of the corresponding phase boundary is $d T / d \bar{\sigma}_{3}=420 \mathrm{~K} \mathrm{GPa}^{-1}$. The diagram in Fig. 6 also indicates that the application of this shear load produces a significant reduction of the otherwise large $t-o 1$ transformation hysteresis. This leads eventually to the appearance of the critical points where the $t-o 1^{+}$and $t$ - $o 1^{-}$equilibrium (Maxwell) lines terminate, as the width of the hysteresis becomes equal to zero. In the area of the diagram adjacent to such critical points the $t$ and $o 1^{+}$, and the $t$ and $o 1^{-}$phases, respectively, can no longer be distinguished. 
The critical points encountered here are similar to the classical thermodynamic singularities known for the gas/ liquid systems, except that in the present case one encounters a "merging" of crystal structures that originally (i.e., for $\bar{\sigma}_{3}=0$ ) had distinct $\mathcal{T}_{3}$ and $\mathcal{O}_{123}$ symmetries. The shear loads play the same role as imperfections in the theory of buckling for structures (or for any bifurcating system): if sufficiently large, they may completely smooth the transition. In the present case, at $p=4 \mathrm{GPa}$ and up to $\bar{\sigma}_{3}=0.025 \mathrm{GPa}$, one should still observe the $t$ - $o 1$ transformation by cycling $T$ in a range between 800 and $900 \mathrm{~K}$; for higher $\bar{\sigma}_{3}$ this transformation should no longer take place, as only one stable phase exists in this region of the diagram (indicated, respectively, by the symbols $t / o 1^{ \pm}$in Fig. 6). ${ }^{37}$

\section{CONCLUSIONS}

While all nonhydrostatic phase equilibria for crystals at finite temperature are fundamentally unstable due to the eventual development of plastic deformation or thermally activated creep, crystalline configurations loaded by shear stresses exist in practice for long times. In these cases of suppressed or strongly delayed relaxation the methods of equilibrium thermodynamics are appropriate, and one can introduce the shear loads as control parameters besides the usual pressure and temperature. This enlargement of the parameter space strongly affects the conventional phase diagrams of materials, and generates a variety of interesting effects, notably the uncoupling of symmetry-related "twin" phases, and the smoothing of symmetry discontinuities around critical points.

In this paper we have studied some of these effects in the case of zirconia, by using the energetic model in Ref. 10. We have explicitly computed several sections of the global nonhydrostatic phase diagram, in which we show both the equilibrium phase boundaries and the coexistence domains for metastable structures. New features emerging as a consequence of the introduction of the shear loads are a number of triple points in the diagrams and critical points, indicating the limits where crystal phases with different symmetries become indistinguishable. Our main result is the prediction that a new orthorhombic phase of $\mathrm{ZrO}_{2}$ (see Appendix B) should be the most stable one for experimentally accessible nonhydrostatic loads in a wide range of temperatures and pressures. The experimental study of zirconia crystals in the suggested domains of shear loads and the parallel ab initio modeling of the new phase should provide proof of the consistency of our approach. $^{38}$

\section{ACKNOWLEDGMENTS}

The work of G.F. was partly supported by TMR Contract No. ERB-FMRX-CT98-0229 of the E.U. and by the Minnesota Supercomputer Institute; L.T. was supported by NSF Grant No. DMS-0102841; G.Z. acknowledges the partial support of the Italian Cofin. MURST2002 "Modelli matematici per la scienza dei materiali" and of Grant No. 127P01 of the Università di Padova.

\section{APPENDIX A}

As explained in detail in Ref. 10, the "minimal" polynomial expansion suitable for the free-energy density $\phi$ in Eq. (6) is

$$
\begin{aligned}
\phi= & \frac{1}{2}\left[\bar{C}_{11} y_{1}^{2}+2 \bar{C}_{12} y_{1} y_{2}+\bar{C}_{22} y_{2}^{2}+\left(C_{11}-C_{12}\right) y_{3}^{2}\right. \\
& \left.+C_{44}\left(y_{4}^{2}+y_{5}^{2}\right)+C_{66} y_{6}^{2}\right]+\frac{1}{2}\left[\left(\bar{C}_{133} y_{1}+\bar{C}_{233} y_{2}\right) y_{3}^{2}\right. \\
& \left.+\left(\bar{C}_{166} y_{1}+\bar{C}_{266} y_{2}\right) y_{6}^{2}+\bar{C}_{344} y_{3}\left(y_{4}^{2}-y_{5}^{2}\right)\right]+\bar{C}_{456} y_{4} y_{5} y_{6} \\
& +\frac{1}{4}\left(D_{3} y_{3}^{4}+2 L y_{3}^{2} y_{6}^{2}+D_{6} y_{6}^{4}\right)+\frac{1}{6}\left(K_{3} y_{3}^{6}+K_{6} y_{6}^{6}\right) . \quad \text { (A1) }
\end{aligned}
$$

In Eq. (A1), (i) $C_{11}, C_{12}, \ldots, C_{66}$, denote the six elastic moduli appearing in the standard tetragonal elastic tensor, ${ }^{29,30}$ and

$$
\begin{gathered}
\bar{C}_{11}=\frac{1}{9}\left[2\left(C_{11}+C_{12}\right)+4 C_{13}+C_{33}\right], \\
\bar{C}_{12}=\frac{2}{3}\left[C_{11}+C_{12}-C_{13}-C_{33}\right], \\
\bar{C}_{22}=2\left[C_{11}+C_{12}-4 C_{13}+2 C_{33}\right] .
\end{gathered}
$$

(ii) $\bar{C}_{I J K}$, for $I, J, K=1, \ldots, 6$, give, in the strain coordinates $y_{I}$, the third-order tetragonal elastic constants ${ }^{39,40}$ considered in the energy function (A1); see Ref. 10 for details. (iii) the moduli $C_{11}-C_{12}$ and $C_{66}$ (which are related to the order parameters $y_{3}$ and $y_{6}$ ) depend on the temperature as follows:

$$
C_{11}-C_{12}=A_{3}\left(T-T_{0}\right), \quad C_{66}=A_{6} T+B_{6},
$$

with $A_{3}>0$ and $A_{6} \geqslant 0$, so that at low pressures the tetragonal phase is stable at high temperatures.

As a result of minimizing the Gibbs free energy function $\phi_{G}$ in Eq. (6) with respect to the non-order-parameter strains, we obtain $y_{4}=y_{5}=0$ and (recall that $-p=\bar{\sigma}_{1}$ )

$$
\begin{gathered}
y_{1}=\frac{1}{\Delta}\left[-\bar{C}_{22} p-\bar{C}_{12} \bar{\sigma}_{2}-\frac{1}{2}\left(H_{3} y_{3}^{2}+H_{6} y_{6}^{2}\right)\right], \\
y_{2}=\frac{1}{\Delta}\left[\bar{C}_{11} \bar{\sigma}_{2}+\bar{C}_{12} p-\frac{1}{2}\left(H_{3}^{\prime} y_{3}^{2}+H_{6}^{\prime} y_{6}^{2}\right)\right],
\end{gathered}
$$

where

$$
\begin{gathered}
\Delta=\bar{C}_{11} \bar{C}_{22}-\bar{C}_{12}^{2}, \\
H_{3}=\bar{C}_{22} \bar{C}_{133}-\bar{C}_{12} \bar{C}_{233}, \quad H_{6}=\bar{C}_{22} \bar{C}_{166}-\bar{C}_{12} \bar{C}_{266}, \\
H_{3}^{\prime}=\bar{C}_{11} \bar{C}_{233}-\bar{C}_{12} \bar{C}_{133}, \quad H_{6}^{\prime}=\bar{C}_{11} \bar{C}_{266}-\bar{C}_{12} \bar{C}_{166} .
\end{gathered}
$$

This gives the Landau energy $\phi_{L}=\psi\left(y_{3}, y_{6}, \bar{\sigma}_{1}, \bar{\sigma}_{2}, T\right)$ $-\bar{\sigma}_{3} y_{3}-\bar{\sigma}_{6} y_{6}$ as in Eq. (8), with 
TABLE II. Candidate structures for the orthorhombic phase $o 2$ of zirconia, satisfying criteria (i)-(iv) in the text. $Z$ gives the number of chemical formulas $\mathrm{ZrO}_{2}$ per unit cell. IR is the irreducible representation of the $t$-phase space group $P 4_{2} / n m c$, giving the transformation mechanism; $\Gamma_{2}^{+}$corresponds to the zero wave vector (Fig. 7 shows the structure arising in this way), while $Z_{i}$ correspond to the wave vector $\left(0,0, \frac{1}{2}\right)$ (Refs. 42 and 46). Origin choice number 2 (i.e., at the inversion center, see Ref. 47) was selected for Pmmn. The last two columns give the Wyckoff positions (Ref. 47) for the $\mathrm{Zr}$ and $\mathrm{O}$ atoms; for instance, $4 c(\times 2)$ means that two sets of Wyckoff positions $4 c$ are occupied. The structure with space group Pnma arises from both the $Z_{3}$ and $Z_{4}$ IRs.

\begin{tabular}{lcccc}
\hline \hline Space Group & $Z$ & ir & $\mathrm{Zr}$ & $\mathrm{O}$ \\
\hline Pmmn & 2 & $\Gamma_{2}^{+}$ & $2 a$ & $2 b(\times 2)$ \\
Pmmn & 4 & $Z_{1}$ & $2 a(\times 2)$ & $2 b(\times 4)$ \\
Pnma & 4 & $Z_{3}, Z_{4}$ & $4 c$ & $4 c(\times 2)$ \\
\hline \hline
\end{tabular}

$$
\begin{aligned}
\psi= & \frac{1}{2}\left(G_{3} y_{3}^{2}+G_{6} y_{6}^{2}\right)+\frac{1}{4}\left(\widetilde{D}_{3} y_{3}^{4}+2 \widetilde{L} y_{3}^{2} y_{6}^{2}+\widetilde{D}_{6} y_{6}^{4}\right) \\
& +\frac{1}{6}\left(K_{3} y_{3}^{6}+K_{6} y_{6}^{6}\right) .
\end{aligned}
$$

The renormalized coefficients can be written:

$$
\begin{gathered}
G_{3}=\left(C_{11}-C_{12}\right)-\frac{1}{\Delta}\left(-H_{3} p+H_{3}^{\prime} \bar{\sigma}_{2}\right), \\
G_{6}=C_{66}-\frac{1}{\Delta}\left(-H_{6} p+H_{6}^{\prime} \bar{\sigma}_{2}\right), \\
\widetilde{D}_{3}=D_{3}-\frac{1}{2 \Delta}\left(\bar{C}_{22} \bar{C}_{133}^{2}-2 \bar{C}_{12} \bar{C}_{133} \bar{C}_{233}+\bar{C}_{11} \bar{C}_{233}^{2}\right), \\
\widetilde{L}=L-\frac{1}{2 \Delta}\left(\bar{C}_{22} \bar{C}_{133} \bar{C}_{166}\right. \\
\left.-\bar{C}_{12}\left(\bar{C}_{133} \bar{C}_{266}+\bar{C}_{166} \bar{C}_{233}\right)+\bar{C}_{11} \bar{C}_{233} \bar{C}_{266}\right), \\
\widetilde{D}_{6}=D_{6}-\frac{1}{2 \Delta}\left(\bar{C}_{22} \bar{C}_{166}^{2}-2 \bar{C}_{12} \bar{C}_{166} \bar{C}_{266}+\bar{C}_{11} \bar{C}_{266}^{2}\right),
\end{gathered}
$$

with $\bar{\sigma}_{3}$ and $\bar{\sigma}_{6}$ given in Eq. (7). The numerical values of the coefficients, taken from Ref. 10, are reported in Table I.

\section{APPENDIX B}

Details about the atomic positions within the $t, o 1$, and $m$ zirconia cells are given in the literature mentioned at the beginning of Sec. II (see Ref. 10 for an illustration). Our approximate model, in which a number of atoms of the zirconia crystal are disregarded (see Fig. 1), can only indicate, for the new $o 2$ phase, the point group (5) and the corresponding skeletal deformation. The configuration of the atoms inside the $o 2$ skeletal cell may then be estimated by some natural criteria that help to identify likely candidate structures: ${ }^{41}$ (i) The space group (SG) of the

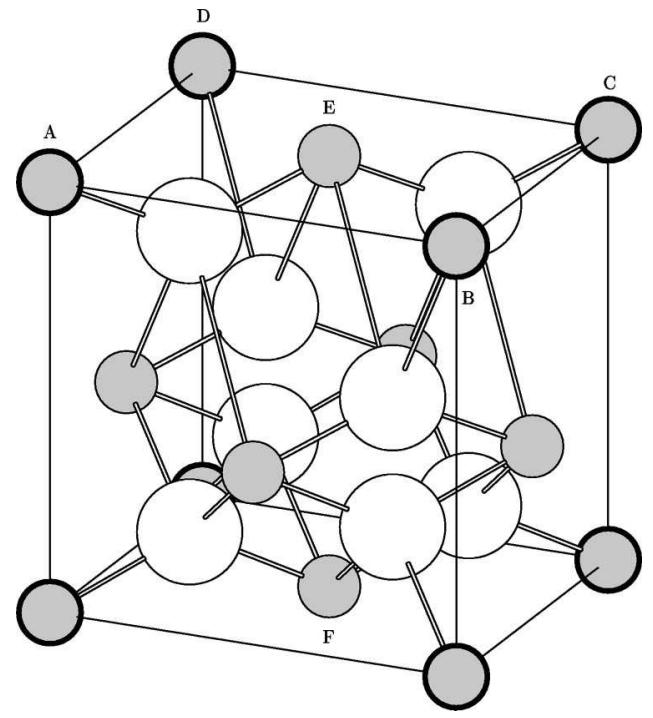

FIG. 7. Representation of a possible candidate structure for the orthorhombic $o 2$ phase of zirconia (originating from mode $\Gamma_{2}^{+}$; see Table II). A skeletal cell as in Fig. 1, with $Z=4$, is shown; $Z r$ atoms are marked in gray, $\mathrm{O}$ atoms in white (the skeletal $\mathrm{Zr}$ atoms are highlighted). The actual unit cell has $Z=2$; the coordination number for the $\mathrm{Zr}$ atoms is 8 . In the basal plane $A B C D E$ of this $o 2$ configuration, the polygon $A B C D$ is a rhombus centered in $E$. The vectors $E B, E C$, and $F E$ are the three mutually orthogonal orthorhombic axes of this lattice.

$o 2$ structure should be a subgroup of the SG $P 4_{2} / n m c$ of the $t$ structure, and a supergroup of the SG $P 2{ }_{1} / c$ of the $m$ structure (this forces the $o 2$ SG to have the orthorhombic holohedry $m m m$ as point group). (ii) There are at most $8 \mathrm{ZrO}_{2}$ chemical units within the $o 2$ skeletal cell, i.e., we take $Z=2,4$, or 8 , for the $o 2$ structure. (iii) The $o 2$ structure is obtained from the $t$ structure through a single normal mode originating from a wave vector on the boundary of the Brillouin zone. Finally, (iv) the strain in the skeletal cell must produce the point group $\mathcal{O}_{1 \pm 2,3}$, i.e., it must be such that two of the orthorhombic axes be along diagonals of the basal square of the skeletal lattice in Fig. 1 (the third one being along the tetragonal axis).

By using the standard methods, ${ }^{42-44}$ and the program ISOTROPY, ${ }^{45}$ we obtain from criteria (i)-(iv) above the three structures listed in Table II (none of which has $Z=8$ ). The simplest such candidate, which originates from mode $\Gamma_{2}^{+}$, is illustrated in Fig. 7; it has $Z=2$ and its unit basis $v_{a}$ coincides with the basis

$$
v_{1}=\frac{1}{2}\left(t_{1}-t_{2}\right), \quad v_{2}=\frac{1}{2}\left(t_{1}+t_{2}\right), \quad v_{3}=t_{3}
$$

of the unit cell of the primitive tetragonal structure of $\mathrm{ZrO}_{2}$, which also has $Z=2$ (we recall that our skeletal tetragonal cell in Fig. 1 has $Z=4$, i.e., twice the volume of such unit cell).

We notice that some possible orthorhombic structures for zirconia have been investigated for instance in Refs. 20 and 48 using density-functional theory, with the aim of assessing 
their relative stability against other tetragonal or cubic configurations adopted by $\mathrm{ZrO}_{2}$ or other oxides or halides. None of these structures, however, are in the list of Table II; establishing the actual nature of the $o 2$ zirconia phase and con- firming its stability at higher pressures, temperatures, and possibly nonhydrostatic loads, needs further careful firstprinciples investigation that is beyond the scope of the present study.
*Electronic address: fadda@aem.umn.edu

†Electronic address: trusk@1ms.polytechnique.fr

†Electronic address: zanzotto@dmsa.unipd.it

${ }^{1}$ B. Budiansky, J. Hutchinson, and J. Lambropoulos, J. Mech. Phys. Solids 19, 337 (1983).

${ }^{2}$ Zirconia Engineering Ceramics, edited by E. H. Kisi (Trans Tech Publ., Ütikon-Zürich, 1998).

${ }^{3}$ P.M. Kelly and L.R.F. Rose, Prog. Mater. Sci. 47, 463 (2002).

${ }^{4}$ S. Block, J.A.H. da Jornada, and G.J. Piermarini, J. Am. Ceram. Soc. 68, 497 (1985).

${ }^{5}$ O. Ohtaka, T. Yamanaka, S. Kume, E. Ito, and A. Navrotsky, J. Am. Ceram. Soc. 74, 505 (1991).

${ }^{6}$ J.M. Léger, P.E. Tomaszewski, A. Atouf, and A.S. Pereira, Phys. Rev. B 47, 14075 (1993).

${ }^{7}$ P. Bouvier, E. Djurado, G. Lucazeau, and T. Le Bihan, Phys. Rev. B 62, 8731 (2000)

${ }^{8}$ O. Ohtaka, H. Fukui, T. Kunisada, T. Fujisawa, K. Funakoshi, W. Utsumi, T. Irifune, K. Kuroda, and T. Kikegawa, Phys. Rev. B 63, 174108 (2001)

${ }^{9}$ L. Truskinovsky and G. Zanzotto, J. Mech. Phys. Solids 50, 189 (2002).

${ }^{10}$ G. Fadda, L. Truskinovsky, and G. Zanzotto, Phys. Rev. B 66, 174107 (2002).

${ }^{11}$ B. Budiansky and L. Truskinovsky, J. Mech. Phys. Solids 41, 1445 (1993).

${ }^{12}$ N. Simha and L. Truskinovsky, Acta Mater. 42, 3827 (1994).

${ }^{13}$ I.-W. Chen, J. Am. Ceram. Soc. 74, 2564 (1991).

${ }^{14}$ P.E. Reyes-Morel and I.-W. Chen, J. Am. Ceram. Soc. 71, 343 (1988).

${ }^{15}$ W.P. Rogers and S. Nemat-Nasser, J. Am. Ceram. Soc. 73, 136 (1990).

${ }^{16}$ D. Baither, M. Bartsch, B. Baufeld, A. Tikhonovsky, A. Foitzik, M. Rühle, and U. Messerschmidt, J. Am. Ceram. Soc. 84, 1755 (2001).

${ }^{17}$ We remark that some of the nonhydrostatic effects described here may be obscured by the onset of plastic flow in zirconia crystals, whose yield stress is estimated to be about $0.8 \mathrm{GPa}$ (see Ref. 16).

${ }^{18}$ E.H. Kisi, C.J. Howard, and R.J. Hill, J. Am. Ceram. Soc. 72, 1757 (1989).

${ }^{19}$ C.J. Howard, E.H. Kisi, R.B. Roberts, and R.J. Hill, J. Am. Ceram. Soc. 73, 2828 (1990).

${ }^{20}$ J.K. Dewhurst and J.E. Lowther, Phys. Rev. B 57, 741 (1998).

${ }^{21}$ S. Fabris, A.T. Paxton, and M.W. Finnis, Phys. Rev. B 61, 6617 (2000).

${ }^{22}$ R.N. Patil and E.C. Subbarao, Acta Crystallogr., Sect. A: Cryst. Phys., Diffr., Theor. Gen. Crystallogr. 26, 535 (1970).

${ }^{23}$ E.C. Subbarao, H.S. Maiti, and K.K. Srivastava, Phys. Status Solidi A 21, 9 (1974).

${ }^{24}$ G.K. Bansal and A.H. Heuer, Acta Metall. 22, 409 (1974).

${ }^{25}$ H. Boysen, F. Frey, and T. Vogt, Acta Crystallogr., Sect. B: Struct. Sci. 47, 881 (1991)
${ }^{26}$ M. Pitteri and G. Zanzotto, Continuum Models for Phase Transitions and Twinning in Crystals (Chapman and Hall, London, 2002).

${ }^{27}$ S.M. Lang, J. Am. Ceram. Soc. 47, 641 (1964).

${ }^{28}$ R.N. Patil and E.C. Subbarao, J. Appl. Crystallogr. 2, 281 (1969).

${ }^{29}$ L. D. Landau and E. M. Lifshits, Theory of Elasticity (Pergamon Press, Oxford, New York, 1986).

${ }^{30}$ M. E. Gurtin, An Introduction to Continuum Mechanics (Academic Press, New York, 1981).

${ }^{31}$ As in our model we disregard a number of atoms in the zirconia crystal (see Fig. 1), here we can only indicate the skeletal deformation and the point group of the new $o 2$ phase of $\mathrm{ZrO}_{2}$. The exact configuration of the disregarded atoms, which would, for instance, allow for an $a b$ initio testing of the stability of such structure, can at this stage only be the object of speculation (see Appendix B).

${ }^{32}$ In this way the loads do not fully distort the crystal, which maintains at least the $\mathcal{M}_{3}$-monoclinic symmetry (see also Sec. IV). If states with $\sigma_{4} \neq 0$ or $\sigma_{5} \neq 0$ are considered, the resulting Landau potential formally coincides with the one in Eq. (A6), but its coefficients would depend also on $\sigma_{4}$ and $\sigma_{5}$, besides $\sigma_{1}=$ $-p$ and $\sigma_{2}$.

${ }^{33}$ For this reason the phase diagrams involving the stress variables $\bar{\sigma}_{3}$ or $\bar{\sigma}_{6}$ are symmetric with respect to the reflection across the $T$ axis.

${ }^{34}$ At high temperatures the metastability domain for the $o 2$ phase reaches into the half plane $p>0$ (see the domain between lines 3 and 9 in Fig. 2), as was already noticed in Ref. 10.

${ }^{35}$ The topological possibility of a $p-T$ diagram with two triple points as in Figs. 2 and 4 was mentioned by P. Toledano and V. Dmitriev, Reconstructive Phase Transitions in Crystals and Quasicrystals (World Scientific, Singapore, 1996). The relevance of this particular pattern for zirconia was shown in Ref. 9.

${ }^{36}$ N. Simha and L. Truskinovsky, in Contemporary Research on the Mechanics and Mathematics of Materials, edited by R. C. Batra and M. F. Beatty (CIMNE, Barcelona, 1996).

${ }^{37}$ We remark that under generic nonhydrostatic loads the symmetry groups of any coexisting structures become immediately indistinguishable. Such different metastable phases still coexist until the shear loads reach critical values (at the critical points) beyond which only one structure is present, any bifurcation being eliminated.

${ }^{38}$ We recall, however, that small amounts of dopants and sample size may strongly affect the stability of the zirconia structures and the phase diagrams; any experimental work should appropriately consider these and other effects that can cloud the predictions of this paper.

${ }^{39}$ J.K. Liakos and G.A. Saunders, Philos. Mag. A 46, 217 (1982).

${ }^{40}$ Elastic, Piezoelectric, and Related Constants of Crystals, edited by R. F. S. Hearmon, Vol. 11 of Landolt-Börnstein, New Series, Group III (Springer-Verlag, Berlin, 1984). 
${ }^{41}$ These conditions lead to the simplest $t-o 2$ transformation mechanisms, restricting considerably the number of possibilities for the $o 2$ structure. However, we remark that neither the orthoI (o 1) nor the $m$ structures of $\mathrm{ZrO}_{2}$ can be obtained in this way from the $t$ phase. [Y. Ishibashi and V. Dvorak, J. Phys. Soc. Jpn. 58, 4211 (1989); K. Negita and H. Takao, J. Phys. Chem. Solids 50, 325 (1989)].

${ }^{42}$ S. C. Miller and W. F. Love, Tables of Irreducible Representations of Space Groups and co-Representations of Magnetic Space Groups (Pruett Press, Boulder, CO, 1967).

${ }^{43}$ I. A. Izyumov and V. N. Syromyatnikov, Phase Transitions and Crystal Symmetry (Kluwer Academic, Dordrecht, 1990).
${ }^{44}$ J.-C. Toledano and P. Toledano, The Landau Theory of Phase Transitions (World Scientific, Singapore, 1987).

${ }^{45}$ H. T. Stokes and D. M. Hatch, Isotropy (1999), http:// www.physics.byu.edu/ stokesh/isotropy.html.

${ }^{46}$ C. J. Bradley and A. P. Cracknell, The Mathematical Theory of Symmetry in Solids; Representation Theory for Point Groups and Space Groups (Clarendon Press, Oxford, 1972).

${ }^{47}$ International Tables for Crystallography, edited by T. Hahn (Reidel, Dordrecht, Boston, 1996), Vol. A.

${ }^{48}$ J.E. Lowther, J.K. Dewhurst, J.M. Léger, and J. Haines, Phys. Rev. B 60, 14485 (1999). 